\title{
Thrombosed Dissection of the Ascending Aorta Complicating Extravasation
}

\author{
Keisuke Morimoto, MD; Toshihiko Tanabe, MD; Satoshi Endoh, MD*; \\ Hiroaki Kuroda, MD**; Shigetsugu Ohgi, MD**; Tohru Mori, MD**
}

\begin{abstract}
This report presents 2 patients with thrombosed dissection of the ascending aorta complicating extravasation. The first case was an 85-year-old male admitted with shock due to cardiac tamponade. Plain computed tomography (CT) demonstrated a dilated ascending aorta without clear evidence of aortic dissection. The second case was a 77-year-old female presenting with shock, in whom an enhanced CT scan demonstrated a dilated ascending aorta and periaortic effusion. However, dissection of the distal ascending aorta was not identified in either case before emergency surgery. In case 1, soon after the bloody pericardial effusion was decompressed during the operation, bleeding from the ascending aorta occurred. A small intimal tear was found in the distal ascending aorta, and in each case the pseudolumen was filled with fresh thrombus. The ascending aorta was replaced. Each patient had an uneventful postoperative recovery. Based on this experience, it is suggested that patients with thrombosed ascending aortic dissection complicating extravasation should undergo early graft replacement. (Jpn Circ J 1999; 63: 652-654)
\end{abstract}

Key Words: Extravasation; Thoracic aorta; Thrombosed dissection

$\mathbf{T}$ hrombosed dissection (TD) of the thoracic aorta has been the focus of recent reports. However, the treatment of patients with TD of the ascending aorta remains controversial. We report 2 patients who underwent successful surgical repair of TD of the ascending aorta complicating extravasation.

\section{Case Reports}

Case 1

An 85-year-old male was admitted with anterior chest discomfort, nausea, and syncope. He was totally healthy until he noted this episode. He had facial edema and dilated neck veins, his pulse rate was 118 beats/min, and systolic blood pressure was $50 \mathrm{mmHg}$. A chest roentgenogram showed a dilated mediastinum. Neither cardiac ischemia nor arrhythmia were demonstrated on electrocardiogram. The serum creatine kinase was not elevated. Other initial laboratory tests did not show remarkable changes. A pericardial effusion was demonstrated by echocardiography, but aortic regurgitation was not detected. The diagnosis of shock due to cardiac tamponade was made. A plain computed tomography (CT) scan demonstrated a dilated ascending aorta without clear evidence of aortic dissection (Fig 1). Further examination was not done due to the unstable hemodynamics. An emergency operation was performed through a median sternotomy, with cannulation of the left femoral artery. Soon after the bloody pericardial effusion was decompressed, bleeding from the ascending aorta occurred. Immediately, circulation was restored via

(Received October 29, 1998; revised manuscript received May 11, 1999; accepted May 12, 1999)

Departments of Cardiovascular Surgery and *Internal Medicine, San-in Rosai Hospital, and **Second Department of Surgery, Tottori University School of Medicine, Yonago, Japan

Mailing address: Keisuke Morimoto, MD, Department of Cardiovascular Surgery, San-in Rosai Hospital, 1-8-1 Kaike Shinden, Yonago 6830002, Japan the femoral artery cannula. Cardiopulmonary bypass (CPB) was started using cannulae in the superior and inferior vena cavae and the left femoral artery. Induction of hypothermia (nasopharyngeal temperature: $17^{\circ} \mathrm{C}$, rectal temperature:
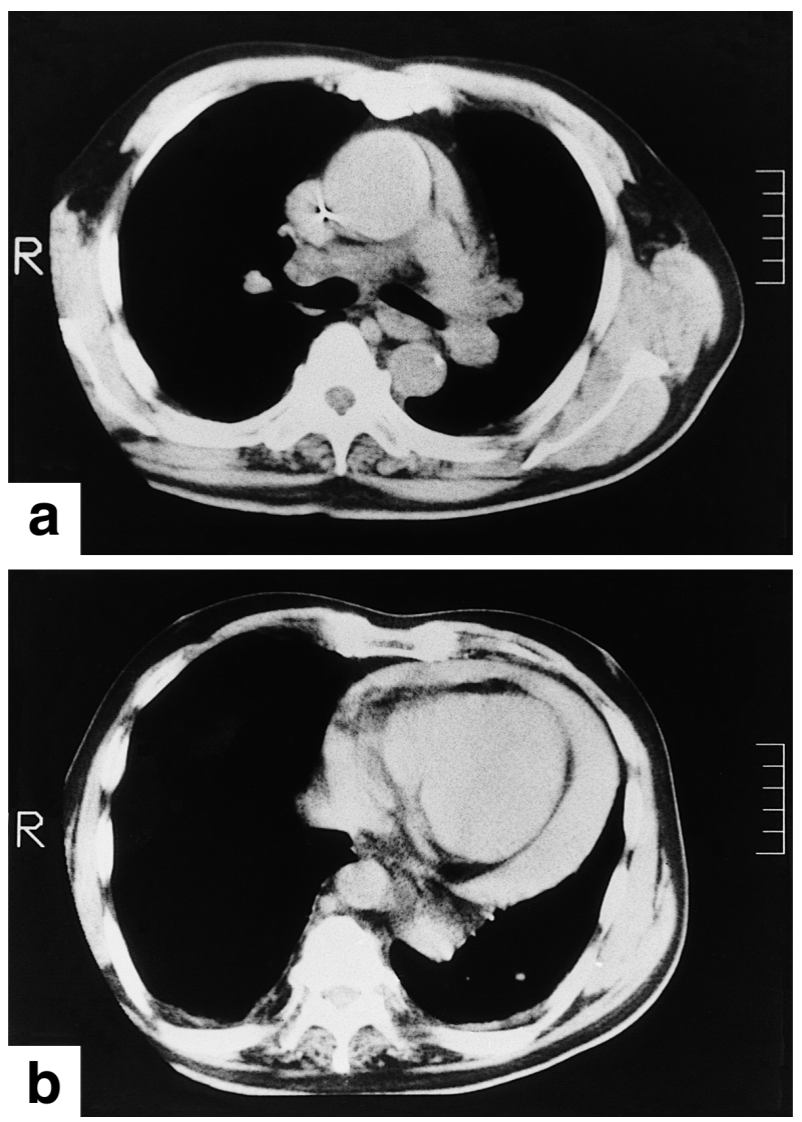

Fig 1. Plain CT demonstrates a dilated ascending aorta. It does not clearly show an aortic dissection (a), but shows cardiac tamponade (b). 

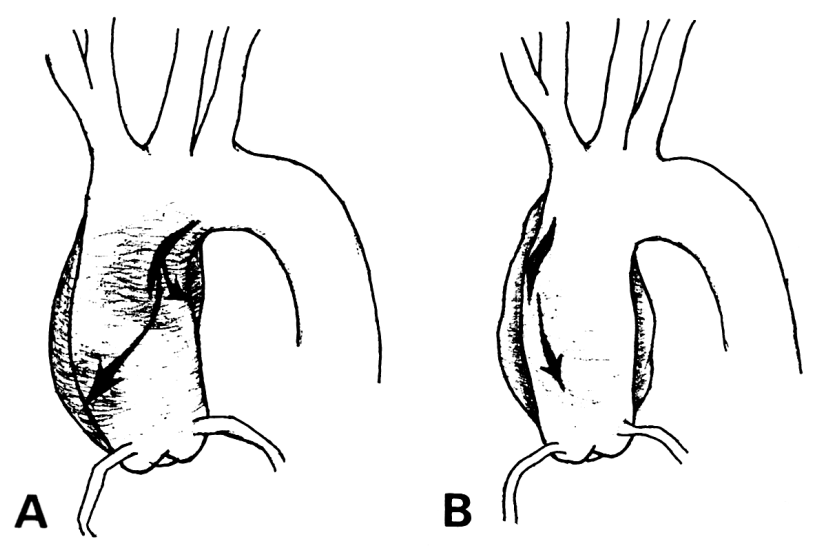

Fig 2. Small intimal tears can be seen in the distal ascending aorta, and the false lumen of the ascending aorta is filled with fresh thrombi. Case 1 (A); Case 2 (B).

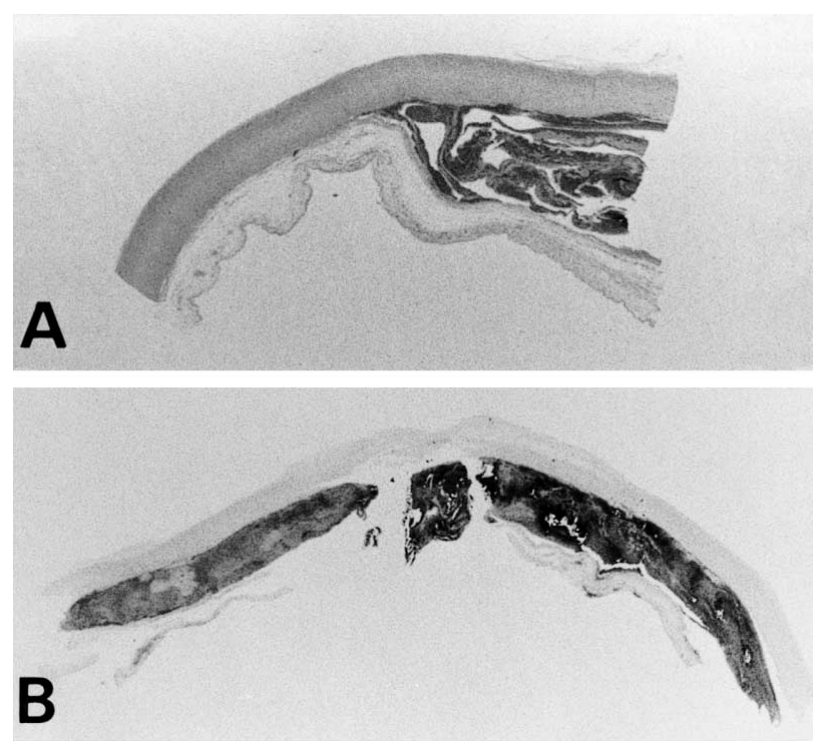

Fig 3. False lumen of the ascending aorta is filled with fresh thrombi. Case 1 (A); Case 2 (B).

$24^{\circ} \mathrm{C}$ ) permitted total circulatory arrest to allow a longitudinal incision of the ascending aorta. Cold blood cardioplegia was administered continuously via the coronary sinus. A small intimal tear was found in the distal ascending aorta, on the left anterolateral side, and the false lumen of the ascending aorta was filled with fresh thrombus (Figs 2A, 3A). However, the site of external rupture was not discovered. The coronary arteries and aortic valve were shown to be intact. Replacement of the ascending aorta using a collagen-impregnated Dacron tube graft was completed using the open aortic anastomosis technique. The patient had an uneventful postoperative course, and is well 2 years after the operation.

\section{Case 2}

A 77-year-old female with a past history of hypertension presented with acute anterior chest pain, syncope, shock, and systolic blood pressure of $70 \mathrm{mmHg}$ with a pulse rate of 110 beats/min. A chest roentgenogram showed a dilated mediastinum. Neither cardiac ischemia nor arrhythmia were demonstrated on electrocardiogram. Laboratory tests

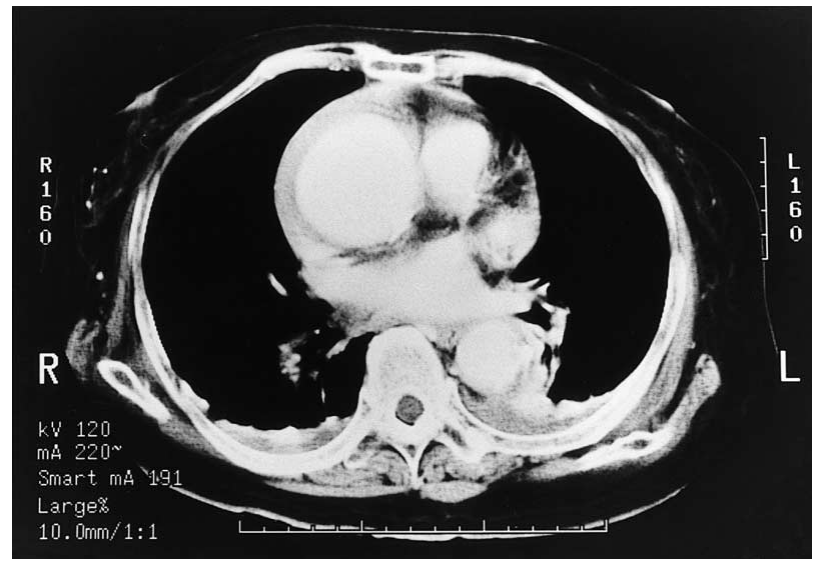

Fig 4. Enhanced CT demonstrates a dilated ascending aorta and periaortic effusion.

yielded the following: white blood cell count of $14.3 \times 10^{3 /}$ $\mu 1$, red blood cell count of $3.54 \times 10^{6} \mu 1$, hemoglobin of 10.3 $\mathrm{g} / \mathrm{dl}$, and platelets of $10.3 \times 10^{4} \mu \mathrm{l}$. Other tests did not show remarkable changes. Enhanced CT scan revealed a dilated ascending aorta and a periaortic effusion (Fig 4). Periaortic effusion was demonstrated by echocardiography, but aortic regurgitation was not detected. An ascending aortic rupture was diagnosed without the identification of a dissection of the ascending aorta. As with case 1, further examination was not done due to the unstable hemodynamics. She underwent emergency surgery via a median sternotomy. Fresh subepicardial and periadventitial hematomas were demonstrated in the ascending aorta. Cannulation for CPB was performed using a single, dual-staged cannula in the right atrium and a smaller cannula in the femoral artery. The ascending aorta was opened under profound hypothermic circulatory arrest (nasopharyngeal temperature: $17^{\circ} \mathrm{C}$, rectal temperature: $20^{\circ} \mathrm{C}$ ). A small intimal tear was found in the distal ascending aorta, on the right lateral side. The false lumen of the ascending aorta was filled with fresh thrombus (Figs 2B, 3B). However, the site of external rupture was not discovered. She also underwent surgical repair and recovered without remarkable postoperative complications. She is alive 10 months after surgery.

\section{Discussion}

In the 2 present cases, the intimal tear was unclear and not detected on the CT images. Discriminating between TD and intramural hematoma (IMH) is difficult clinically or preoperatively. IMH is a diagnosis of exclusion and presents as localized hemorrhage into the wall of aorta in the absence of bona fide aortic dissection, intimal tear, or penetrating atherosclerotic ulcer! Nienaber et al think intramural hemorrhage may be considered an ominous precursor of overt aortic dissection and have reported 12 patients with intramural hemorrhage of the ascending aorta? Of these, 7 underwent surgical intervention with $2(28 \%)$ postoperative deaths. The remaining 5 cases received medical treatment. Of these 5 cases, 4 (80\%) died between $24 \mathrm{~h}$ and 7 days after diagnosis. Nienaber et al mentioned that early surgical repair should be considered for all patients with ascending aortic involvement (type A intramural hemorrhage). They conclude that IMH is associated with a clinical profile and prognosis similar to classic dissection and that the outcome of IMH of the ascending aorta appears 
favorable only with immediate surgical repair. Treatment of patients with TD may be similar to that of patients with IMH.

Yamada et al have reported 14 cases of acute aortic dissection without an intimal tear? Six of these patients had involvement of the ascending aorta (type A). All patients were treated medically with periodic CT or magnetic resonance imaging (MRI) follow-up. Two of the 6 patients (33\%) died of type A dissection (one died of intrathoracic hemorrhage following pericardiocentesis, and the other of ventricular fibrillation). Both patients had pericardial effusion, and pericardial effusion was noted in 3 patients with type A dissection.

Robbins et al have reported 3 cases of IMH of the thoracic aorta with the involvement of the ascending aorta! All of them ultimately required surgical intervention, and 2 $(67 \%)$ died. One had a pericardial tap to evacuate a bloody pericardial effusion, but on day 5 after the acute event, the ascending aorta ruptured. The patient could not be weaned from CPB after emergency graft replacement of the ascending aorta. Robbins et al conclude that patients with ascending aorta/arch IMH should probably undergo early graft replacement!

The surgical indication of TD of the ascending aorta remains controversial. Further studies are needed before treatment guidelines can be established. Because pericardiocentesis is considered to be the standard treatment for patients suffering from cardiac tamponade, it is not surprising that it has been used as an interim treatment for patients with cardiac tamponade complicating aortic dissection while they await definitive surgical repair4 However, Isselbacher et al have reported that in patients with cardiac tamponade complicating aortic dissection, pericardiocentesis can be harmful rather than beneficial, and have suggested that cardiac tamponade should be considered a surgical emergency? Patients with IMH of the ascending aorta complicating extravasation have a particularly high risk of death and so pericardiocentesis should be avoided until early surgical repair of the aorta is performed with intraoperative drainage of periaortic or pericardiac effusion. We consider that the unstable patients with type A acute thrombosed aortic dissection complicating extravasation, such as the present cases, should undergo early aggressive surgical intervention with only CT imaging, without angiography.

In the present cases, replacement of the ascending aorta was completed using the technique of open aortic anastomoses $^{6}$ under profound hypothermic circulatory arrest. Reinforcement of the aortic wall with double layers of Teflon felt was effective for preventing excessive bleeding. The 2 patients underwent successful surgical repair of the TD of the ascending aorta complicating extravasation. Thus we also suggest that patients with ascending TD complicating extravasation should undergo early graft replacement, without pericardiocentesis.

\section{References}

1. Robbins RC, McManus RP, Mitchell RC, Latter DR, Moon MR Olinger GN, et al: Management of patients with intramural hematoma of the thoracic aorta. Circulation 1993; 88(Suppl II): II-1-II-10

2. Nienaber CA, von Kodolitsch Y, Petersen B, Loose R, Helmchen U, Haverich A, et al: Intramural hemorrhage of the thoracic aorta: Diagnostic and therapeutic implications. Circulation 1995; 92: $1465-1472$

3. Yamada T, Tada S, Harada J: Aortic dissection without intimal rupture: Diagnosis with MR imaging and CT. Radiology 1988; 168: $347-352$

4. Garcia-Jimenez A, Torres AP, Lopez GM, Dieguez IA, Alba CMB Cardiac tamponade by aortic dissection in a hospital without cardiothoracic surgery. Chest 1993; 104: 290-291

5. Isselbacher EM, Cigarroa JE, Eagle KA: Cardiac tamponade complicating proximal aortic dissection: Is pericardiocentesis harmful? Circulation 1994; 90: 2375-2378

6. Livesay JJ, Cooly DA, Duncan JM, Ott DA, Walker WE, Reul GJ: Open aortic anastomosis: Improved results in the treatment of aneurysms of the aortic arch. Circulation 1982; 66(Suppl I): I-122-I-127 